\title{
Elasticity Tensors in Nematic Liquid Crystals
}

\section{Amelia Carolina Sparavigna}

${ }^{1}$ Department of Applied Science and Technology, Politecnico di Torino, Italy

\begin{abstract}
The paper is discussing the contributions to the free energy of elastic distortions in a nematic liquid crystal. These contributions are here given by tensors, which are represented by means of the components of the director, the unit vector indicating the local average alignment of molecules, and by Kronecker and Levi-Civita symbols. The paper is also discussing the elasticity of the second order and its contribution in threshold phenomena.
\end{abstract}

Keywords: Liquid Crystals, Nematics, Elasticity, Continuum Theories

\section{Introduction}

The equation of Oseen-Frank, which is providing the free energy density in nematic liquid crystals [1,2], is representing the part of that energy density coming from an elastic deformation of the bulk of the material. In such approach, the terms depending on the deformations are multiplied by the three elastic constants $\left(K_{11}, K_{22}, K_{33}\right)$ of "splay", "twist" and "bend". After, to this energy density, Nehring and Saupe added two terms having elastic constants of "mixed splay-bend" $\left(K_{13}\right)$ and of "saddle- splay" $\left(K_{24}\right)$ [3] . These terms, which have $K_{13}, K_{24}$ as elastic constants, are equivalent to contributions to the surface free energy of the material, because they are divergences of some vector functions of the director. These contributions to surface energy are relevant for nematic cells subjected to weak anchoring conditions [4-6].

The elastic constants previously mentioned are constants that multiply some scalar functions obtained from the director, the unit vector giving the local average alignment of the molecules, and from its derivatives, in the form of rotors and divergences. In this paper, we will discuss how the terms of the free energy density in nematic liquid crystals can be described by tensors, and how these tensors can be represented using the director components and the Kronecker and Levi-Civita symbols. This paper will also discuss the terms of the second order and their role in threshold phenomena $[7,8]$.

\section{The elastic deformation of a nematic}

In an ideal nematic, the molecules are oriented, in average, along the director, the macroscopic unit vector $\mathbf{n}$ [9]. This alignment of molecules along a common direction is proper of the nematic phase, which, in this manner, is characterized by an orientational order. This order disappears in the isotropic phase. To describe the nematic phase, a tensor order parameter is given as:

$$
Q_{i j}=Q(T)\left(n_{i} n_{j}-\frac{1}{3} \delta_{i j}\right)
$$

In (1), $i$ and $j$ are indices of the Cartesian frame of reference; $n_{i}$ are the director components and $\delta_{i j}$ the Kronecker symbol. Scalar $Q$ depends on the temperature and goes to zero in the isotropic phase. Tensor $Q_{i j}$ can be a function of the position vector $\mathbf{r}$. Let us remember that in the macroscopic approach of the continuum mechanics, this position vector does not describe the positions of the single microscopic molecules.

If the order parameter is depending on position $\mathbf{r}$, then we need to assume this dependence through the director as follow:

$$
Q_{i j}(\mathbf{r})=Q(T)\left(n_{i}(\mathbf{r}) n_{j}(\mathbf{r})-\frac{1}{3} \delta_{i j}\right)
$$

In the continuum approach, the deformation of the bulk of the material is described by means of three fundamental deformations [9]. They are the deformations of splay, twist and bend. The features of them are the following. In the splay, we have div $\boldsymbol{n}$ $\neq 0$. In the bend, we have that rot $\boldsymbol{n}$ is perpendicular to $\mathbf{n}$. Then, in the twist deformation, rot $\boldsymbol{n}$ is parallel to $\mathbf{n}$.

This article is published under the terms of the Creative Commons Attribution License 4.0 Author(s) retain the copyright of this article. Publication rights with Alkhaer Publications.

Published at: http://www.ijsciences.com/pub/issue/2016-07/

DOI: 10.18483/ijSci.1117; Online ISSN: 2305-3925; Print ISSN: 2410-4477 
Oseen and Frank demonstrated that the density of the free energy given by distortions in the second order of $\mathbf{n}$ is represented by the three deformations:

$$
\begin{aligned}
f_{d} & =\frac{1}{2}\left[K_{1}(\operatorname{div} \mathbf{n})^{2}+K_{2}(\mathbf{n} \cdot \operatorname{rot} \mathbf{n})^{2}\right. \\
& \left.+K_{3}(\mathbf{n} \times \operatorname{rot} \mathbf{n})^{2}\right]
\end{aligned}
$$

In (3), $K_{1}$ is the elastic constant of splay, $K_{2}$ of twist and $K_{3}$ of bend. Since it is possible to have deformations of pure splay, or of twist or bend, each of these elastic constants must be positive. If it were not so, the undistorted deformation would not be that having the minimum energy.

The elastic constants $K_{i}$ have the dimensions of a force, as we can see, using pure dimensional analysis, from Eq. 3 where we have $\left[\right.$ Energy $\left./ \mathrm{L}^{3}\right]=[K]\left[\mathrm{L}^{-2}\right]$, and $\mathrm{L}$ is a length. Then $[$ Energy $/ \mathrm{L}]=[K]$. Let us note that $\mathbf{n}$ is a dimensionless quantity. $K$ is of the order of $U / a$, where $U$ means a typical energy of interaction between molecules $(U \sim 2 \mathrm{Kcal} / \mathrm{mole})$ and $a$ is the molecular dimension of length $(a \sim 14 \AA \dot{A})$. So, we have [10]:

$$
K \cong \frac{1.4 \times 10^{-13} \mathrm{erg}}{1.4 \times 10^{-7} \mathrm{~cm}}=10^{-6} \mathrm{dyne}
$$

For the liquid crystal PAA about $120^{\circ} \mathrm{C}$, it is $K_{1}=0.7$ $10^{-6}$ dyne, $K_{2}=0.4310^{-6}$ dyne, $K_{3}=1.710^{-6}$ dyne [10].

Assuming $K_{1}=K_{2}=K_{3}=K$, we obtain the oneelastic-constant approximation:

$$
F_{d}=\frac{1}{2} K\left[(\operatorname{div} \mathbf{n})^{2}+(\operatorname{rot} \mathbf{n})^{2}\right]
$$

To determine the contribution to the free energy coming from the elastic deformation, Oseen and Frank used the following approach [9]. Let us assume the $z$-axis along the direction of director $\mathbf{n}$, the $x$-axis perpendicular to the director and $y$-axis perpendicular to $x$ - and $z$-axes, oriented according the right-hand rule.

We can distinguish six elemental distortions, linked to the director variation. Therefore, the deformations of splay, bend and twist can be described by means of six derivatives:

$$
\begin{aligned}
& \partial n_{y} / \partial y=a_{5} ; \partial n_{x} / \partial x=a_{1} \\
& \partial n_{x} / \partial z=a_{3} ; \partial n_{y} / \partial z=a_{6} \\
& \partial n_{x} / \partial y=a_{2} ; \partial n_{y} / \partial x=a_{4}
\end{aligned}
$$

For small deformations, we have:

$$
\begin{aligned}
& n_{z} \approx 1 \\
& n_{x} \approx a_{1} x+a_{2} y+a_{3} z \\
& n_{y} \approx a_{4} x+a_{5} y+a_{6} z
\end{aligned}
$$

Then, we can write the density of the free energy coming from the distortion of the nematic according to the Hooke law, as a quadratic function of the deformation:

$$
\begin{aligned}
& g=K_{i} a_{i}+\frac{1}{2} K_{i j} a_{i} a_{j} \\
& K_{i j}=K_{j i} ; i, j=1,2, \ldots, 6
\end{aligned}
$$

In (8), there are six elastic parameters $K_{i}$ and 36 elastic parameters $K_{i j}$. Anyway, using symmetries these numbers of parameter are reduced. For instance, the cylindrical symmetry of the nematic liquid crystal implies invariance for rotation about $\mathrm{z}$-axis. So the energy is invariant during such rotation. Then, because of invariance for transformation $x^{\prime}=y, y^{\prime}=$ $\mathrm{x}, \mathrm{z}^{\prime}=\mathrm{z}$, we have only two independent $K_{\mathrm{i}}$ parameters $\left(K_{1}, K_{2}\right)$ and five independent $K_{i j}$ parameters $\left(K_{11}\right.$ $\left.K_{22}, K_{33}, K_{24}, K_{12}\right)$.

Moreover, we have the condition $\mathbf{n}=-\mathbf{n}$, which is telling that the director direction does not influence the features of the medium. In this case, it is necessary that transformation $z^{\prime}=-z, y^{\prime}=-y, x^{\prime}=-x$, does not change the deformation of the threedimensional structure. So we need $K_{1}$ and $K_{13}$ being zero. Also the mirror symmetry exists, and then, according to transformation $\mathrm{x}^{\prime}=\mathrm{x}, \mathrm{y}^{\prime}=-\mathrm{y}, \mathrm{z}^{\prime}=\mathrm{z}$, we find $K_{2}$ and $K_{12}$ being zero.

In this manner, in the elastic energy density of nematics we have just four coefficients: $K_{11}, K_{22}, K_{33}$ and $K_{24}$. However, the term multiplied by coefficient $\mathrm{K}_{24}$ is not proper of the bulk energy, because it is a contribution to the surface energy of the material [4]. Consequently, the Frank equation for the density of the elastic energy is given by the following elemental deformations [9]: 


$$
\begin{aligned}
g & =\frac{1}{2}\left[K_{11}\left(a_{1}+a_{5}\right)^{2}+K_{22}\left(a_{2}-a_{4}\right)^{2}\right. \\
& \left.+K_{33}\left(a_{3}+a_{6}\right)^{2}\right]
\end{aligned}
$$

We can write (9) using rotor and divergence of director:

$$
\begin{aligned}
g & =\frac{1}{2}\left[K_{11}(\operatorname{div} \mathbf{n})^{2}+K_{22}(\mathbf{n} \cdot \operatorname{rot} \mathbf{n})^{2}\right. \\
& \left.+K_{33}(\mathbf{n} \times \operatorname{rot} \mathbf{n})^{2}\right]
\end{aligned}
$$

In the case of cholesteric nematics, $K_{2} \neq 0$. An additional contribution given by $K_{2} \mathbf{n} \cdot \operatorname{rotn}$, is required in the second term of (10). So, instead of $K_{22}(\mathbf{n} \cdot \operatorname{rot} \mathbf{n})^{2}$, we have

$$
\begin{aligned}
& g=\frac{1}{2}\left[K_{11}(\operatorname{div} \mathbf{n})^{2}\right. \\
& +K_{22}\left(\mathbf{n} \cdot \operatorname{rot} \mathbf{n}+K_{2} / K_{22}\right)^{2} \\
& \left.+K_{33}(\mathbf{n} \times \operatorname{rot} \mathbf{n})^{2}\right]
\end{aligned}
$$

In fact, squaring the second term, the contribution of $\left(K_{2} / K_{22}\right)^{2}$ is not relevant, because it does not depend on the deformation. The ratio $\mathrm{q}_{0}=K_{2} / K_{22}$ is the modulus of the wave-vector of the cholesteric helical structure, having pitch $\mathrm{P}_{0}=2 \pi / \mathrm{q}_{0}$. In the case $K_{2} \neq 0$, the equilibrium configuration of the cholesteric nematic is that having a spontaneous twisted deformation.

3. Elastic energy density, given by the derivatives $n_{i, j}=\partial n_{i} / \partial x_{j}$

Following the Oseen - Frank approach, let us being more general. If the director $\mathbf{n}$ does not depend on position, the liquid crystal is undistorted and the free energy density is supposed equal to a certain quantity represented by $f_{0}$, which is a quantity that does not change if the liquid crystals is subjected to a deformation.

If $\mathbf{n}=\mathbf{n}(\mathbf{r})$, the nematic is deformed. Let $f$ being the density of elastic energy which is created in the material. We have then that $n_{i, j}=\partial n_{i} / \partial x_{j}$ are different from zero. Let us suppose that these derivatives of $\mathbf{n}$ are enough for describing the distorted nematic, and then:

$$
f=f\left(n_{i, j}\right)
$$

If these derivatives are small, it is possible to consider a series in $n_{i, j}$, so that:

$$
f=f_{o}+E_{i j} n_{i, j}+\frac{1}{2} K_{i j k l} n_{i, j} n_{k, l}
$$

In (129, the components of tensors $\boldsymbol{E}$ and $\boldsymbol{K}$ are given by:

$$
E_{i j}=\left(\frac{\partial f}{\partial n_{i, j}}\right)_{o} ; K_{i j k l}=\left(\frac{\partial^{2} f}{\partial n_{i, j} \partial n_{k, l}}\right)_{o}
$$

These quantities are evaluated with respect the undistorted configuration, which is also that having the minimum energy. In (12), we have used Einstein notation which implies summation on a set of indices; for instance $E_{i j} n_{i, j}=\sum_{i, j} E_{i j} n_{i, j}$.

Let us write $\boldsymbol{E}$ and $\boldsymbol{K}$, using combinations made by $\mathbf{n}$, and the Kronecker $\delta_{i j}$ and Levi-Civita $\varepsilon_{i j k}$ symbols. Since in a nematic, $\mathbf{n}$ and $\mathbf{- n}$ are equivalent, each term in (2) must be even in $\mathbf{n}$. Let us remember that, for what is concerning the Kronecker symbol, it is $\delta_{i j}=1$, if $i=j$. It is $\delta_{i j}=0$ if $i \neq j$. In the case of the Levi-Civita symbol, we have $\varepsilon_{i j k}=1$ if $i, j, k$ are in cyclic order; $\varepsilon_{i j k}=-1$ if $i, j, k$ are not in cyclic order. Then, we have $\varepsilon_{i j k}=0$ in the other cases. Moreover, the cross product of two vectors $\mathbf{a}$ and $\mathbf{b}$ can be written as $\mathbf{a} \times \mathbf{b}=\varepsilon_{k i j} a_{i} b_{j} \mathbf{e}_{k}$, where $\mathbf{e}_{k}$ is a unit vector of the three unit vectors representing a Cartesian frame of reference.

In the case of the rotor of the director, we can write it as:

$$
\operatorname{rot} \mathbf{n}=\varepsilon_{k i j} n_{i, j} \mathbf{e}_{k}
$$

Let us consider tensor $\boldsymbol{E}$. We can represent its components as:

$$
E_{i j}=E_{1} n_{i} n_{j}+E_{2} \delta_{i j}+E_{3} n_{k} \varepsilon_{k i j}
$$

But $E_{1}=E_{2}=0$, because the nematic is not polar. Moreover:

$$
\begin{aligned}
E_{i j} n_{i, j} & =E_{3} n_{k} \varepsilon_{k i j} n_{i, j} \\
& =-E \mathbf{n} \cdot \operatorname{rot} \mathbf{n}
\end{aligned}
$$


This is a pseudoscalar, being the nematic helicity $[11,12]$. Since the energy is a scalar, $\mathbf{n} \cdot \operatorname{rot} \mathbf{n}$ can be present in it, when this contribution is squared or if the nematic is a cholesteric nematic. In this case, the material is spontaneously showing a deformed configuration of the fundamental state having the minimum energy.

For tensor $\boldsymbol{K}$ we have $K_{i j k l}=K_{k l i j}$, because it is present in the term $K_{i j k l} n_{i, j} n_{k, l}$; so we can write:

$$
K_{i j k l}=C_{123}+C_{4567}
$$

In (16), we have:

$$
\begin{aligned}
& C_{123}=K_{1} n_{i} n_{j} n_{k} n_{l}+K_{2} n_{i} n_{j} \delta_{k l} \\
& +K_{2}^{*} n_{k} n_{l} \delta_{i j}+K_{3} n_{i} n_{k} \delta_{j l}+K_{3}^{*} n_{j} n_{l} \delta_{i k} \\
& C_{4567}=K_{4} n_{i} n_{l} \delta_{j k}+K_{4}^{*} n_{j} n_{k} \delta_{i l}+K_{5} \delta_{i j} \delta_{k l} \\
& +K_{6} \delta_{i k} \delta_{j l}+K_{7} \delta_{i l} \delta_{j k}
\end{aligned}
$$

Since $n_{i} n_{i}=1$, we can see that in (16) just some terms survive. They are:

$$
\begin{aligned}
K_{3}^{*} n_{j} n_{l} \delta_{i k} n_{i, j} n_{k, l} & =K_{3}^{*} n_{j} n_{l} n_{i, j} n_{i, l} \\
& =K_{3}^{*}(\mathbf{n} \times \operatorname{rot} \mathbf{n})^{2}
\end{aligned}
$$

$$
\begin{aligned}
& K_{5} \delta_{i j} \delta_{k l} n_{i, j} n_{k, l}=K_{5}(\operatorname{div} \mathbf{n})^{2} \\
& K_{6} \delta_{i k} \delta_{j l} n_{i, j} n_{k, l}+K_{7} \delta_{i l} \delta_{j k} n_{i, j} n_{k, l} \\
& =K_{6} n_{k, l} n_{k, l}+K_{7} n_{l, j} n_{j, l}
\end{aligned}
$$

Let us demonstrate the first (I). Using the Levi-Civita symbol for the cross product, we have:

$$
(\mathbf{n} \times \operatorname{rot} \mathbf{n})_{k}=\varepsilon_{k i j} n_{i} \varepsilon_{j l m} n_{l, m}
$$

Note that we have to sum on $j$. So, the following relation exists:

$$
\varepsilon_{k i j} \varepsilon_{j l m}=\delta_{k l} \delta_{i m}-\delta_{k m} \delta_{i l}
$$

Therefore:

$$
\begin{aligned}
& (\mathbf{n} \times \operatorname{rotm})_{k}=n_{i} n_{k, i}-n_{i} n_{i, k} \\
& =n_{i} n_{k, i}-\frac{1}{2} \partial_{k}\left(n_{i} n_{i}\right)=n_{i} n_{k, i}
\end{aligned}
$$

We used the fact that the director is a unit vector and then the derivative of its modulus is zero. (II) and (III) contain factor $2 n_{i} n_{i, j}=\partial_{j}\left(n_{i} n_{i}\right)=0$. Then, all the terms we are re-writing in the following formula are null, so as their sum:

$$
\begin{aligned}
& K_{1} n_{i} n_{j} n_{k} n_{l} n_{i, j} n_{k, l}+K_{2} n_{i} n_{j} \delta_{k l} n_{i, j} n_{k, l}+K_{2}^{*} n_{k} n_{l} \delta_{i j} n_{i, j} n_{k, l}+K_{3} n_{i} n_{k} \delta_{j l} n_{i, j} n_{k, l}+K_{4} n_{i} n_{l} \delta_{j k} n_{i, j} n_{k, l} \\
& +K_{4}^{*} n_{j} n_{k} \delta_{i l} n_{i, j} n_{k, l}=K_{1} n_{i} n_{j} n_{k} n_{l} n_{i, j} n_{k, l}+K_{2} n_{i} n_{j} n_{i, j} n_{k, k}+K_{2}^{*} n_{k} n_{l} n_{i, i} n_{k, l}+K_{3} n_{i} n_{k} n_{i, j} n_{k, j} \\
& +K_{4} n_{i} n_{l} n_{i, j} n_{j, l}+K_{4}^{*} n_{j} n_{k} n_{l, i} n_{k, l}=0
\end{aligned}
$$

The surviving terms which are contributing to the elastic energy are:

$$
K_{3}^{*}(\mathbf{n} \times \operatorname{rot} \mathbf{n})^{2}+K_{5}(\operatorname{div} \mathbf{n})^{2}+K_{6} n_{k, j} n_{k, j}+K_{7} n_{l, j} n_{j, l}
$$

We have [7]:

$$
\begin{aligned}
& n_{k, j} n_{k, j}=n_{k, j} n_{j, k}+(\mathbf{n} \cdot \operatorname{rot} \mathbf{n})^{2}+(\mathbf{n} \times \operatorname{rot} \mathbf{n})^{2} \\
& n_{k, j} n_{j, k}=(\operatorname{div} \mathbf{n})^{2}-\operatorname{div}(\mathbf{n} \operatorname{div} \mathbf{n}+\mathbf{n} \times \operatorname{rot} \mathbf{n})
\end{aligned}
$$

Finally, we have: 


$$
\begin{aligned}
& \left(K_{5}+K_{6}+K_{7}\right)(\operatorname{div} \mathbf{n})^{2}+K_{6}(\mathbf{n} \cdot \operatorname{rot} \mathbf{n})^{2}+\left(K_{3}^{*}+K_{6}\right)(\mathbf{n} \times \operatorname{rot} \mathbf{n})^{2} \\
- & \left(K_{6}+K_{7}\right) \operatorname{div}(\mathbf{n} \operatorname{div} \mathbf{n}+\mathbf{n} \times \operatorname{rot} \mathbf{n})
\end{aligned}
$$

We can change the symbols for the coefficients, to see that this expression is that of the free energy density proposed by Oseen - Frank, with the saddle-splay term too.

$$
\begin{aligned}
f_{\text {Frank }} & =\frac{1}{2} K_{11}(\operatorname{div} \mathbf{n})^{2}+\frac{1}{2} K_{22}(\mathbf{n} \cdot \operatorname{rot} \mathbf{n})^{2}+\frac{1}{2} K_{33}(\mathbf{n} \times \operatorname{rot} \mathbf{n})^{2} \\
& -\left(K_{22}+K_{24}\right) \operatorname{div}(\mathbf{n} \operatorname{div} \mathbf{n}+\mathbf{n} \times \operatorname{rot} \mathbf{n})
\end{aligned}
$$

As previously told, $K_{11}, K_{22}, K_{33}$ e $K_{24}$ are the elastic constants of splay, twist, bend, and saddle-splay. The last term in (21), if we consider the Gauss theorem, is a contribution to the surface energy. Then, the density energy of the bulk is depending on the three elastic constants of splay, twist and bend. If we consider also the helicity, the free energy becomes:

$$
\begin{aligned}
f & =f_{o}-E \mathbf{n} \cdot \operatorname{rot} \mathbf{n}+\frac{1}{2} K_{11}(\operatorname{div} \mathbf{n})^{2}+\frac{1}{2} K_{22}(\mathbf{n} \cdot \operatorname{rot} \mathbf{n})^{2}+\frac{1}{2} K_{33}(\mathbf{n} \times \operatorname{rot} \mathbf{n})^{2} \\
& -\left(K_{22}+K_{24}\right) \operatorname{div}(\mathbf{n} \operatorname{div} \mathbf{n}+\mathbf{n} \times \operatorname{rot} \mathbf{n})
\end{aligned}
$$

If we have not splay and bend, and only twist is present, Equation (22) is minimized by:

$$
\frac{E}{K_{22}}=\mathbf{n} \cdot \operatorname{rot} \mathbf{n}
$$

Then, in the case we have helicity $(E \neq 0)$, the configuration of the director which is minimizing the energy is a distorted one. As previously told, this happens in the cholesteric liquid crystals. From now on, we assume a nematic having $E=0$.

\section{The analysis of Nehring and Saupe}

Let $\mathbf{n}$ be the director and $n_{i, j}, n_{i, j k}$ the derivatives of first and second order. Let us suppose a free energy density depending on these derivatives $f=f\left(n_{i, j}, n_{i, j k}\right)$ and represent it as a series of $n_{i, j}$ and $n_{i, j k}$ :

$$
f=f_{0}+K_{i j k l} n_{i, j} n_{k, l}+L_{i j k} n_{i, j k}+M_{i j k l m n} n_{i, j k} n_{l, m n}+N_{i j k l m} n_{i, j} n_{k, l m}
$$

Being tensor $\boldsymbol{L}$ multiplying derivatives of the second order, it must be:

$$
L_{i j k}=L_{i k j}
$$

As we have done before, let us represent the components of tensors $\boldsymbol{L}, \boldsymbol{M}$ and $\boldsymbol{N}$, using $\mathbf{n}, \delta_{i j}$ and $\varepsilon_{i j k}$. Let us start from $\boldsymbol{L}$ and determine its contribution to energy. Being $\mathbf{n}$ and $-\mathbf{n}$ equivalent, the terms different from zero are:

$$
L_{i j k}=L_{1} n_{i} n_{j} n_{k}+L_{2} n_{i} \delta_{j k}+L_{3}\left(n_{j} \delta_{i k}+n_{k} \delta_{i j}\right)
$$

Then:

$$
L_{i j k} n_{i, j k}=L_{1} n_{i} n_{j} n_{k} n_{i, j k}+L_{2} n_{i} n_{i, j j}+L_{3} n_{j} n_{k, k j}+L_{3} n_{k} n_{j, j k}
$$


Again, let us remember that $n_{i} n_{i}=1$, and therefore:

$$
n_{i} n_{i, j}=0 \quad ; \quad n_{i, k} n_{i, j}=-n_{i} n_{i, j k}
$$

Using (27), the first term in (26) becomes $n_{i} n_{j} n_{k} n_{i, j k}=-\left(n_{k} n_{i, k}\right)^{2}$. The second becomes $n_{i} n_{i, j j}=-n_{i, j} n_{i, j}$, The third is equivalent to:

$$
n_{i} n_{j, j i}=\left(n_{i} n_{j, j}\right)_{, i}-n_{i, i} n_{j, j}=\operatorname{div}(\mathbf{n} \operatorname{div} \mathbf{n})-\left(n_{j, j}\right)^{2}
$$

Equation (26) can be written as

$$
L_{i j k} n_{i, j k}=-\left[L_{1}\left(n_{k} n_{i, j}\right)^{2}+L_{2}\left(n_{i, j}\right)^{2}+2 L_{3}\left(n_{j, j}\right)^{2}\right]+2 L_{3} \operatorname{div}(\mathbf{n} \operatorname{div} \mathbf{n})
$$

We have already found the first three terms of (28), when we have discussed the tensor $\boldsymbol{K}$. These terms can be added to those previously given. But in (28) we have a new term which is contributing to the surface term, like that having as elastic constant $K_{24}$. This term is usually written with the constant $K_{13}$, defined as the elastic constant of splaybend $[13,14]$.

Supposing $\boldsymbol{M}=0$ and $\boldsymbol{N}=0,(24)$ becomes:

$$
f=f_{0}+L_{i j k} n_{i, j k}+K_{i j k l} n_{i, j} n_{k, l}
$$

Therefore, after renormalizing the constants:

$$
\begin{gathered}
f_{N S}=f_{0}+\frac{1}{2}\left[K_{11}(\operatorname{div} \mathbf{n})^{2}+K_{22}(\mathbf{n} \cdot \operatorname{rot} \mathbf{n})^{2}+K_{33}(\mathbf{n} \times \operatorname{rot} \mathbf{n})^{2}\right]+ \\
-\left(K_{22}+K_{24}\right) \operatorname{div}(\mathbf{n} \operatorname{div} \mathbf{n}+\mathbf{n} \times \operatorname{rot} \mathbf{n})+K_{13} \operatorname{div}(\mathbf{n} \operatorname{div} \mathbf{n})
\end{gathered}
$$

This is the free energy density of Nehring-Saupe [3]. However, we have also the terms coming from tensors $\boldsymbol{M}$ and $\boldsymbol{N}$, to consider as sources of elastic constants, it they are different from zero. To dealt with them we need a second order analysis.

\section{Second order analysis}

We have seen in the previous section, that the free energy density, as given by Nehring and Saupe, including the term with elastic constant $K_{13}$, is originated from tensors $\boldsymbol{K}$ and $\boldsymbol{L}$. However, other terms exist that we have not yet discussed. To analyse them let us follow the approach given in [7]. In this reference, the free energy density is supposed a function of deformations as:

$f=f\left(n_{i, j}, n_{i, j k}\right)$

That is, we assume as deformation sources, the firstand second-order derivatives of the director, generalizing the approach of the previous section.

The virtual variation $\delta f$ of the density of the free energy $f$, close to equilibrium, can be described to the second order as:

$$
\delta f=\frac{\delta f}{\delta n_{i, j}} \delta n_{i, j}+\frac{\delta f}{\delta n_{i, j k}} \delta n_{i, j k}=\lambda_{i j} \delta n_{i, j}+\mu_{i j k} \delta n_{i, j k}
$$


In (31), we find the variations of the first- and second-order derivatives of director $\mathbf{n}$. In the linear theory of elasticity, in (31) we have to consider just the derivatives of the first-order, neglecting those of higher order. If the second-order is involved too, tensors $\lambda, \boldsymbol{\mu}$ must be expanded in terms of the sources of deformation, $n_{i, j}$ and $n_{i, j k}$. Therefore:

$$
\left\{\begin{aligned}
\lambda_{i j} & =\lambda_{i j}^{0}+A_{i j k l} n_{k, l}+B_{i j k l m} n_{k, l m}+C_{i j k l m p} n_{k, l} n_{m, p}+ \\
& +D_{i j k l m p q} n_{k, l m} n_{p, q}+H_{i j k l m p q r} n_{k, l} n_{m, p} n_{q, r} \\
\mu_{i j k} & =\mu_{i j k}^{0}+M_{i j k l m} n_{l, m}+N_{i j k l m p} n_{l, m p}+O_{i j k l m p q} n_{l, m} n_{p, q}
\end{aligned}\right.
$$

In (32) $\lambda^{\circ}, \boldsymbol{\mu}^{\circ}$ are tensors depending just on the components $n_{i}$ but not dependent on $n_{i, j}$ and $n_{i, j k}$, The same for tensors $\boldsymbol{A}, \boldsymbol{B}, \boldsymbol{C}, \boldsymbol{D}, \boldsymbol{H}, \boldsymbol{M}, \boldsymbol{N}$ and $\boldsymbol{O}$.

Using (32) in (31), we see that only $\lambda^{\circ}$ is described by terms of the first order, whereas $\boldsymbol{A}, \boldsymbol{\mu}^{\circ}$ have terms of the second order, $\boldsymbol{B}, \boldsymbol{C}, \boldsymbol{M}$ of the third and $\boldsymbol{D}, \boldsymbol{H}, \boldsymbol{O}$ of the fourth order.

We could ask ourselves if, in (32), it is also necessary a term of the form $F_{i j k l m p} n_{k, l m p}$, which is of the same order of $\boldsymbol{D}$ and $\boldsymbol{H}$. In the approach we are following here, this term is not coming from the sources of deformation we are considering. And then, we do not include it, or other similar to it, in (32). The criterion is that $\lambda_{i j}$ and $\mu_{i j k}$ are expanded only in terms of the deformation sources, as defined in (31).

Let us take into account the general property of the mixed second-order derivatives of continuous function $g$ :

$$
\frac{\partial^{2} g}{\partial n_{i, j} \partial n_{k, l m}}=\frac{\partial^{2} g}{\partial n_{k, l m} \partial n_{i, j}}
$$

Equation (33) ensures that [7]:

$$
B_{i j k l m}=M_{k l m i j} \quad ; \quad D_{i j k l m p q}=O_{k l m i j p q}
$$

Moreover, from symmetry considerations [7]:

$$
\begin{aligned}
& A_{i j k l}=A_{k l i j} ; C_{i j k l m p}=C_{i j m p k l}=C_{k l i j m p} ; D_{i j k l m p q}=D_{p q k l m i j} \\
& H_{i j k l m p q r}=H_{k l i j m p q r}=H_{i j m k l q r}=H_{i j k l q r m p}=H_{q r k l m p i j}=H_{m p k l i j q r}
\end{aligned}
$$

Using (32) in (31), according to (33') and (34), we have [7]:

$$
\begin{aligned}
f & =\lambda_{i j}^{0} n_{i, j}+\mu_{i j k}^{0} n_{i, j k}+\frac{1}{2} A_{i j k l} n_{i, j} n_{k, l}+B_{i j k l m} n_{i, j} n_{k, l m}+\frac{1}{3} C_{i j k l m p} n_{i, j} n_{k, l} n_{m, p} \\
& +\frac{1}{2} D_{i j k l m p q} n_{i, j} n_{k, l m} n_{p, q}+\frac{1}{4} H_{i j k l m p q r} n_{i, j} n_{k, l} n_{m, p} n_{q, r}+\frac{1}{2} N_{i j k l m p} n_{i, j k} n_{l, m p}
\end{aligned}
$$

Usually, common nematics are not polar or chiral and then, in (35), odd terms in $\mathbf{n}$ and helicity $\mathbf{n} \cdot \operatorname{rot} \mathbf{n}$ are not present. Therefore, for common nematics tensors $\lambda^{\circ}, \boldsymbol{B}$ e $\boldsymbol{C}$ are null.

Then, we have only: 


$$
\begin{aligned}
f & =\mu_{i j k}^{0} n_{i, j k}+\frac{1}{2} A_{i j k l} n_{i, j} n_{k, l}+\frac{1}{2} D_{i j k l m p q} n_{i, j} n_{k, l m} n_{p, q} \\
& +\frac{1}{4} H_{i j k l m p q r} n_{i, j} n_{k, l} n_{m, p} n_{q, r}+\frac{1}{2} N_{i j k l m p} n_{i, j k} n_{l, m p}
\end{aligned}
$$

Let us expand tensors in $\left(35^{\prime}\right)$, using $\left(n_{i}, \delta_{i j}, \varepsilon_{i j k}\right)$. After expanding the tensors, the terms that survive are (given in covariant form, that in using divergence and rotor):

$$
\begin{aligned}
& f_{A}=A_{1}(\operatorname{div} \mathbf{n})^{2}+A_{2}(\mathbf{n} \cdot \operatorname{rot} \mathbf{n})^{2}+A_{3}(\mathbf{n} \times \operatorname{rot} \mathbf{n})^{2} \\
& +A_{4} \operatorname{div}(\mathbf{n} \operatorname{div} \mathbf{n}+\mathbf{n} \times \operatorname{rot} \mathbf{n}) \\
& \quad f_{\mu}=\mu_{1}(\operatorname{div} \mathbf{n})^{2}+\mu_{2}(\mathbf{n} \cdot \operatorname{rot} \mathbf{n})^{2}+\mu_{3}(\mathbf{n} \times \operatorname{rot} \mathbf{n})^{2} \\
& +\mu_{4} \operatorname{div}(\mathbf{n} \operatorname{div} \mathbf{n})-\mu_{2} \operatorname{div}(\mathbf{n} \operatorname{div} \mathbf{n}+\mathbf{n} \times \operatorname{rot} \mathbf{n})
\end{aligned}
$$

If we have only $n_{i, j}$ as source of deformation, (35') becomes:

$$
f=\lambda_{i j}^{0} n_{i, j}+\frac{1}{2} A_{i j k l} n_{i, j} n_{k, l}
$$

In this case, the term with $K_{13}$ does not appear, in agreement with the elastic theory of the first order as given by Oseen-Frank. Therefore, if we consider $K_{13}$, and also $K_{24}$, we can ask ourselves what are the other terms we need to consider too, to have the expression of $f$ coherent to the second order approach given in [7]. In we assume (36a) and (36b), we have the wellknown splay, twist and bend distortions coming from terms due to tensors $\boldsymbol{\mu}^{\circ}$ and $\boldsymbol{A}$. However, we have many terms from tensors $\boldsymbol{N}, \boldsymbol{D}$ and $\boldsymbol{H}$ (many of them are null because of the parity of $\mathbf{n}$ and pseudoscalarity of $(\mathbf{n} \cdot \operatorname{rot} \mathbf{n}))$. Let us note also that some terms are coming from both $\boldsymbol{N}$ and $\boldsymbol{D}$, or from $\boldsymbol{D}$ and $\boldsymbol{H}$, or from the three tensors at the same time.

In the three Tables I-III given after the References we show the contributions to the density of the free energy of a nematic liquid crystal coming from $N, D$ and $\boldsymbol{H}$. In the first of the three tables, the terms are coming from $\boldsymbol{N}$. In the second table we have contributions coming from $\boldsymbol{D}$, and those marked by $*$ are shared with $\boldsymbol{N}$. In the third table we have the contributions to the density of the free energy coming from $\boldsymbol{H}$ (terms marked by $*$ are shared with $\boldsymbol{D}$, whereas those marked by $* *$ are shared with $N$ and D).

\section{Second-order elasticity close to a threshold}

If we use for a nematic liquid crystal the theory of the first-order elasticity, we have only three constants to deal with. Whereas, if we use the theory of the second order, we have a quite larger number of elastic constant. Then, the use of such theory turns out to be unworkable. In fact, the elasticity of the second order can become quite simple, when it is involved in a threshold phenomenon, when the nematic is changing its configuration from an undistorted to a distorted one.

Let us remember that the most common experimental condition to study the nematic is that of creating a cell composed by two plane-parallel glass slides, spaced a few microns, in which the nematic is inserted by capillarity. The two inner surfaces of the cell may have the same or different treatments, in order to induce the desired nematic configuration (planar, homeotropic or hybrid). The cell can be placed in a thermostat with appropriate optical windows for the observation under polarized microscopy of the phases or of the material configurations.

Let us consider a flat deformation of the nematic, that is, one which is obtained for example in a cell with opposite conditions, homeotropic on one of the walls and planar on to the other (this is the so-called hybrid HAN-cell. We can use a frame of reference $(x, z)$ with the origin on the wall with homeotropic anchoring, the $\mathrm{x}$-axis parallel to that wall, and the $\mathrm{z}$-axis perpendicular to it.

Figure 1 shows how the molecules can be in the HAN cell. They lie in the xz-plane, and are anchored parallel to the upper wall and perpendicular to the 
lower wall. In the intermediate part of the cell, the director forms an angle different from $0^{\circ}$ and from $90^{\circ}$, with respect the $\mathrm{z}$-axis.

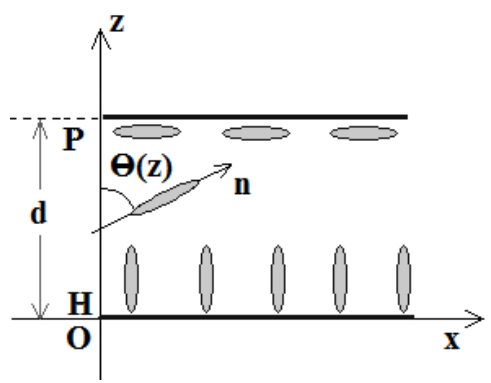

Figure 1: Configuration of the molecules at the walls of a HAN cell in strong anchoring conditions.
Locally, the director $\mathbf{n}$ is given by:

$\mathbf{n}=\mathbf{i} \sin (\Theta)+\mathbf{k} \cos (\Theta)$

In (37), $\Theta(z)$ is the angle shown in the Figure 1. Vectors $\mathbf{i}$ and $\mathbf{k}$ are the unit vectors of axes.

If we imagine to decrease the cell thickness $d$, the deformed configuration becomes unstable and the cell, if we suppose that the planar anchoring is stronger than the homeotropic one, assumes the planar configuration. Or, if we assume the homeotropic anchoring stronger, the cell assumes the homeotropic configuration. The transition that occurs in the HAN cell, when we are reducing the thickness of the liquid crystal, is from a distorted configuration to the undistorted planar $(\mathrm{P})$ or homeotropic $(\mathrm{H})$ configuration (see Figure

2).
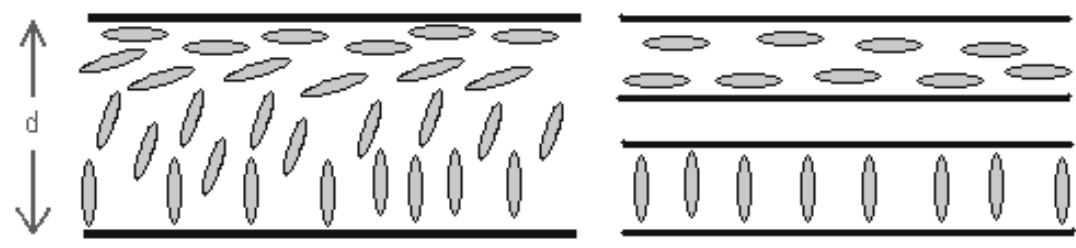

Figure 2: Decreasing the cell thickness, we can see the distorted HAN configuration becoming a planar or homeotropic undistorted configuration shown on the right of the image.

In order to describe the occurrence of a limit for the mechanical stability of the HAN cell, due to the decrease of the cell thickness $d$, the free energy density must be given as a function of angle $\Theta$. Close the threshold of the passage from the HAN configuration to the undistorted $\mathrm{P}$ or $\mathrm{H}$ configurations, we have $\Theta$ changing its value. However, the value of this angle depends on the positon of the director in the cell, that is, on $\mathrm{z}$. So let us take as the parameter for studying the problem, the maximum angle assumed by the director with respect to $\mathrm{z}$-axis and call it $\Theta_{\max }$. If the planar anchoring is strong, this angle can also be of $90^{\circ}$. In fact, we can think the angle $\Theta$ as a function of $z$, and therefore as a function $\Theta=\Theta_{\max } g(z / d)$.

Now, let us consider some terms from Tables I-III, and write them in covariant form [7]:

$$
\begin{aligned}
& n_{i, i j} n_{k, k j}=(\operatorname{grad} \operatorname{div} \mathbf{n})^{2} ; n_{i, j j} n_{i, k k}=\left(\nabla^{2} \mathbf{n}\right)^{2} \\
& n_{i, j} n_{i, j} n_{k, l} n_{k, l}=\left(\mathbf{n} \cdot \nabla^{2} \mathbf{n}\right)^{2} ; n_{j} n_{k} n_{m} n_{p} n_{i, j} n_{i, k} n_{l, m} n_{l, p}=(\mathbf{n} \times \operatorname{rot} \mathbf{n})^{4}
\end{aligned}
$$

Using (37), these terms become:

$$
\begin{gathered}
(\operatorname{grad} \operatorname{div} \mathbf{n})^{2}=\cos ^{2} \Theta(\dot{\Theta})^{4}+\sin 2 \Theta(\dot{\Theta})^{2} \ddot{\Theta}+\sin ^{2} \Theta(\ddot{\Theta})^{2} \\
\left(\nabla^{2} \mathbf{n}\right)^{2}=(\dot{\Theta})^{4}+(\ddot{\Theta})^{2}(39 b) \\
\left(\mathbf{n} \cdot \nabla^{2} \mathbf{n}\right)^{2}=(\dot{\Theta})^{4}
\end{gathered}
$$




$$
(\mathbf{n} \times \operatorname{rot} \mathbf{n})^{4}=\cos ^{4} \Theta(\dot{\Theta})^{4}
$$

In (39a)-(39d), we used $\dot{\Theta}=d / d z$. Let us suppose a transition from HAN to H configuration. Near the threshold, $\Theta_{\max } \rightarrow 0$, and then the contributions of the smallest order in $\Theta_{\max }$ are coming from the term $(\ddot{\Theta})^{2}$ in $(39 b)$. Let us stress that $(\ddot{\Theta})^{2}$ is of the second order in $\Theta_{\max }$, whereas the other contributions vanish more rapidly, when $\Theta_{\max }$ goes to zero. As a consequence, we have an additional term to the free energy density which can be written as:

$$
K^{*}\left(\nabla^{2} n\right)^{2} \cong K^{*}(\stackrel{\bullet}{\Theta})^{2}
$$

In the framework of the theory of elasticity here discussed, only $K^{*}$ is the new elastic constant of the second order which survives in a threshold phenomenon. In this case, the free energy density becomes:

$$
\begin{aligned}
f= & \frac{1}{2}\left\{K_{11}(\operatorname{div} \mathbf{n})^{2}+K_{22}(\mathbf{n} \cdot \operatorname{rot} \mathbf{n})^{2}+K_{33}(\mathbf{n} \times \operatorname{rot} \mathbf{n})^{2}\right\}+ \\
& +K_{13} \operatorname{div}(\mathbf{n} \operatorname{div} \mathbf{n})-\left(K_{22}+K_{24}\right) \operatorname{div}(\mathbf{n} \operatorname{div} \mathbf{n}+\mathbf{n} \times \operatorname{rot} \mathbf{n})+K^{*}\left(\nabla^{2} \mathbf{n}\right)^{2}
\end{aligned}
$$

Let us note that the first five elastic constants have dimensions of energy divided by length; the elastic constant $K^{*}$ is an energy times a length. Let us evaluate its order as made for (4). We have $[$ Energy $]\left[\mathrm{L}^{-3}\right]=\left[K^{*}\right]\left[\mathrm{L}^{-4}\right]$, where $\mathrm{L}$ is a length. Then [Energy][L] $=\left[K^{*}\right]$ and then the order of $K^{*}$ is $U \cdot a$. If $U$ is the typical energy of molecular interaction and $a$ the typical length of molecules, we have:

$$
K^{*} \cong 1.4 \times 10^{-13} \mathrm{erg} \cdot 1.4 \times 10^{-7} \mathrm{~cm} \cong 2 \times 10^{-20} \mathrm{erg} \cdot \mathrm{cm}=2 \times 10^{-20} \mathrm{dyne} \cdot \mathrm{cm}^{2}
$$

Let us consider the deformation as $\Theta=\Theta_{\max } g(z / d)$. Let $\varsigma=z / d$ be the dimensionless variable. Deformation are of the following orders:

$$
\begin{aligned}
& \dot{\Theta}=\frac{d}{d z} \Theta=\frac{d \varsigma}{d z} \frac{d \Theta}{d \varsigma}=\frac{1}{d} \frac{d \Theta}{d \varsigma} \approx \frac{1}{d} \Theta_{\max } g_{, \varsigma} \\
& \ddot{\Theta}=\frac{d^{2}}{d z^{2}} \Theta=\frac{d \varsigma}{d z} \frac{d}{d \varsigma}\left(\frac{1}{d} \frac{d \Theta}{d \varsigma}\right) \approx \frac{1}{d^{2}} \Theta_{\max } g_{, \varsigma \varsigma}
\end{aligned}
$$

We can define $\xi$ the dimensionless derivative (slope) of $g$ with respect to $\varsigma$, that is $g_{, \varsigma}$, and the dimensionless curvature $\kappa$ of this function as approximated by $g, \varsigma \varsigma$. We will have:

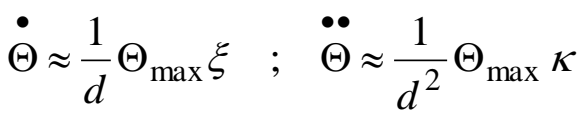

Then: 


$$
f \approx \frac{K}{d^{2}} \Theta_{\max }^{2} \xi^{2}+\frac{K^{*}}{d^{4}} \Theta_{\max }^{2} \kappa^{2}
$$

We can evaluate the thickness $d$ of the cell for which the term with $K^{*}$ becomes relevant. This happens when the two terms in (42), are comparable:

$$
\frac{K \xi^{2}}{d^{2}} \Theta_{\max }^{2} \approx \frac{K^{*} \kappa^{2}}{d^{4}} \Theta_{\max }^{2} \rightarrow d^{2} \approx \frac{K^{*}}{K} \frac{\kappa^{2}}{\xi^{2}}
$$

We have that the thickness $d$ for which $\mathrm{K}^{*}$ is relevant depends on the square ratio between curvature and slope of the deformation, a ratio which is depending on the anchoring conditions of the cell. As a conclusion we can tell that, in certain anchoring conditions of the cell, the term with elastic constant $K^{*}$ cannot be neglected in evaluating the threshold value of the cell thickness.

\section{References}

1) Oseen, C. W. (1933). The theory of liquid crystals. Transactions of the Faraday Society, 29(140), 883-899. DOI: http://dx.doi.org/10.1039/tf9332900883

2) Frank, F. C. (1958). I. Liquid crystals. On the theory of liquid crystals. Discussions of the Faraday Society, 25, 19-28. DOI: http://dx.doi.org/10.1039/df9582500019

3) Nehring, J., \& Saupe, A. (1972). Calculation of the elastic constants of nematic liquid crystals. J. Chem. Phys. 56, 5527 5529 DOI: http://dx.doi.org/10.1063/1.1677071

4) Sparavigna, A., Lavrentovich, O. D., \& Strigazzi, A. (1994). Periodic stripe domains and hybrid-alignment regime in nematic liquid crystals: Threshold analysis. Physical Review E, $\quad 49(2), \quad 1344 . \quad$ DOI: http://dx.doi.org/10.1103/physreve.49.1344

5) Sparavigna, A., Komitov, L., Stebler, B., \& Strigazzi, A. (1991). Static splay-stripes in a hybrid aligned nematic layer. Molecular Crystals and Liquid Crystals 207(1), 265-280. DOI: http://dx.doi.org/10.1080/10587259108032105

6) Sparavigna, A., Komitov, L., Lavrentovich, O. D., \& Strigazzi, A. (1992). Saddle-splay and periodic instability in a hybrid aligned nematic layer subjected to a normal magnetic field. Journal de Physique II, 2(10), 1881-1888. DOI: http://dx.doi.org/10.1051/jp2:1992241

7) Barbero, G., Sparavigna, A., \& Strigazzi, A. (1990). The structure of the distortion free-energy density in nematics: second-order elasticity and surface terms. Il Nuovo Cimento D, 12(9), 1259-1272. http://dx.doi.org/10.1007/bf02450392

8) Sparavigna, A., Komitov, L., \& Strigazzi, A. (1991). Hybrid Aligned Nematics and second order elasticity. Physica Scripta, 43(2), 210. DOI: http://dx.doi.org/10.1088/0031 8949/43/2/017

9) Stephen, M. J., \& Straley, J. P. (1974). Physics of liquid crystals. Reviews of Modern Physics, 46(4), 617-704. DOI http://dx.doi.org/10.1103/revmodphys.46.617

10) De Gennes, P. G., \& Prost, J. (1995). The Physics of Liquid Crystals, Clarendon Press. ISBN: 0198517858 , 9780198517856

11) Sparavigna, A. C. (2012). Distortional Lifshitz vectors and helicity in nematic free energy density. arXiv preprint arXiv:1207.2918.

12) Sparavigna, A. C. (2013). Distortional Lifshitz vectors and Helicity in nematic free energy density, International Journal of $\quad$ Sciences 07(2013):54-59. DOI http://dx.doi.org/10.18483/ijsci.211

13) Lavrentovich, O. D., \& Pergamenshchik, V. M. (1995) Patterns in thin liquid crystal films and the divergence ("surfacelike") elasticity. International Journal of Modern Physics B, 9, 2389-2437. DOI http://dx.doi.org/10.1142/9789812831101_0008

14) Pergamenshchik, V. M., Subacius, D., \& Lavrentovich, O. D. (1997). $\mathrm{K}_{13}$-induced deformations in a nematic liquid crystal: Experimental test of the first-order theory. Molecular Crystals and Liquid Crystals, 292(1), 25-37. DOI http://dx.doi.org/10.1080/10587259708031918 

1) $n_{i, j j} n_{k, k i}$
2) $n_{i, i j} n_{k, k j}$
3) $n_{i, j k} n_{i, j k}$
4) $n_{i, j k} n_{j, i k}$
5) $n_{i, j j} n_{i, k k}$
6) $n_{i} n_{j} n_{k, k l} n_{l, i j}$
7) $n_{i} n_{j} n_{k, l l} n_{k, i j}$
8) $n_{i} n_{j} n_{k, k i} n_{l, l j}$
9) $n_{i} n_{j} n_{k, i l} n_{l, j k}$
10) $n_{i} n_{j} n_{k, i l} n_{k, j l}$
11) $n_{i} n_{j} n_{k} n_{l} n_{m, i j} n_{m, k l}$

\section{Table I}
1) $n_{i} n_{j, i} n_{k, k} n_{j, l l}$
2) $n_{i} n_{j, i} n_{k, k} n_{l, l j}$
4) $n_{i} n_{j, i} n_{k, l} n_{j, k l}$
5) $n_{i} n_{j, i} n_{k, l} n_{l, j k}$
7) $n_{i} n_{j, i} n_{k, j} n_{k, l l}$
8) $n_{i} n_{j, k} n_{k, l} n_{l, l i}$
10) $n_{i} n_{j, j} n_{k, l} n_{l, i k}$
11) $n_{i} n_{j, k} n_{k, l} n_{j, i l}$
13) $n_{i} n_{j, j} n_{k, k} n_{l, l i}$
14) $n_{i} n_{j, k} n_{l, k} n_{l, i j}$
16) $n_{i} n_{j, k} n_{j, k} n_{l, l i}$ *
17) $n_{i} n_{j, i k} n_{j, l} n_{k, i l}$ *
19) $n_{i} n_{j} n_{k} n_{l, l} n_{m, i} n_{m, j k}$
20) $n_{i} n_{j} n_{k} n_{l, i} n_{m, k} n_{m, j l}$
22) $n_{i} n_{j} n_{k} n_{l, i} n_{l, k} n_{m, m j}$ *
23) $n_{i} n_{j} n_{k} n_{l, i} n_{l, m} n_{m, j k}$ *

3) $n_{i} n_{j, i} n_{k, l} n_{k, j l}$

6) $n_{i} n_{j, i} n_{k, j} n_{l, k k}$

9) $n_{i} n_{j, k} n_{l, l} n_{j, k i}$

12) $n_{i} n_{j, k} n_{k, l} n_{l, i j}$

15) $n_{i} n_{j, i} n_{j, k} n_{k, l l}$ *

18) $n_{i} n_{j, k} n_{j, i} n_{k, l l}$ *

\section{Table II}
1) $n_{i, i} n_{j, j} n_{k, k} n_{l, l}$
2) $n_{i, j} n_{j, i} n_{k, l} n_{l, k}$
3) $n_{i, j} n_{j, i} n_{k, k} n_{l, l}$
4) $n_{i, j} n_{j, k} n_{k, l} n_{l, i}$
5) $n_{i, i} n_{j, k} n_{l, j} n_{k, l}$
7) $n_{i, i} n_{j, k} n_{k, l} n_{j, l}$ *
8) $n_{i, j} n_{j, k} n_{k, l} n_{i, l}$ *
10) $n_{i, j} n_{j, i} n_{k, l} n_{k, l}$ *
11) $n_{i, j} n_{k, j} n_{i, l} n_{k, l} * *$
13) $n_{i} n_{j} n_{k, i} n_{l, m} n_{l, j} n_{k, m}$ *
14) $n_{i} n_{j} n_{k, k} n_{l, m} n_{l, i} n_{m, j}$ *
6) $n_{i, i} n_{j, j} n_{k, l} n_{k, l}$ *
16) $n_{i} n_{j} n_{k, j} n_{k, i} n_{l, l} n_{m, m}$ *
17) $n_{i} n_{j} n_{k, i} n_{k, j} n_{l, m} n_{l, m}$ *
9) $n_{i, j} n_{k, i} n_{l, j} n_{k, l}$ *
12) $n_{i, j} n_{i, j} n_{k, l} n_{k, l} * *$
19) $n_{i} n_{j} n_{k, l} n_{k, m} n_{m, i} n_{l, j} * *$
20) $n_{i} n_{j} n_{k, l} n_{k, l} n_{m, i} n_{m, j} * *$
15) $n_{i} n_{j} n_{k, l} n_{m, i} n_{k, j} n_{l, m} *$
18) $n_{i} n_{j} n_{k, j} n_{k, i} n_{l, m} n_{m, l} *$
13) $n_{i} n_{j} n_{k} n_{l} n_{m, i} n_{m, j} n_{p, k} n_{p, l} * *$

\section{Table III}

Tables I-III: These three Tables are giving the contributions to the density of the free energy of a nematic liquid crystal coming from tensors $\boldsymbol{N}, \boldsymbol{D}$ and $\boldsymbol{H}$, respectively. In the second Table, contributions coming from $\boldsymbol{D}$ marked by * are those shared with $\boldsymbol{N}$. In the third Table, contributions to the free energy density coming from $\boldsymbol{H}$, marked by *, are shared with $\boldsymbol{D}$, whereas those marked by ** are shared with $\boldsymbol{N}$ and $\boldsymbol{D}$. 